\title{
Research on the Inheritance and Sustainable Development of National Intangible Cultural Heritage Gambiered Canton Gauze
}

\author{
Zicheng Pan ${ }^{1, *}$ Caifu Liao ${ }^{1}$ Xiudan Lin ${ }^{1}$ Ruijiong Lin ${ }^{1}$ \\ ${ }^{1}$ School of Management, University of Electronic Science and Technology of China, Zhongshan Institute, Zhongshan, \\ Guangdong 528402, China \\ *Corresponding author. Email: 1501669111@qq.com
}

\begin{abstract}
As a national intangible cultural heritage (ICH) in China, Gambiered Canton Gauze (GCG) has encountered difficulties in the process of its craft inheritance and sustainable development, and empirical research on the inheritance and development of ICH is rare. Therefore, this study takes citizens as the research object, and explores the influencing factors in the inheritance and development of GCG from the perspective of the public through questionnaire survey. The results show that: policy management factors, cultural factors, economic factors and living inheritance have a significant positive impact on the craft inheritance and sustainable development of GCG; the internal and external live inheritance have partial mediating effect.
\end{abstract}

Keywords: Intangible cultural heritage, Gambiered Canton Gauze, living inheritance, sustainable development

\section{INTRODUCTION}

Gambiered Canton Gauze (GCG) is a specialty of Shunde, Foshan, Guangdong province, a kind of silk fabric made from extractions from plants and minerals. As this insect-repellent, anti-microbial material feels comfortable and pleasantly cool, is beneficial to the skin, is easy to wash and does not wrinkle, it possesses high practical, technological, cultural and economic value. This leads to the nickname of "soft gold" in ancient times because it was only a choice for wealthy families. As GCG is unique in production and difficult to be transplanted, which reflects the regional characteristics of Lingnan, it was listed as a national intangible cultural heritage in China in 2008. Due to the complicated production techniques, time-consuming and laborious process, high cost of cloth and labor, as well as the lack of technical talents, few product categories on the market, low visibility and insufficient publicity, there are many obstacles in the skill inheritance and sustainable development of GCG. Therefore, from the perspective of the public, this paper focuses on the development of GCG through questionnaire survey, with the aim to find out the factors influencing the inheritance and development of GCG as intangible cultural heritage, and relevant measures and suggestions are put forward in the paper.

\section{LITERATURE REVIEW}

\subsection{Inheritance and Development of Intangible Cultural Heritage}

Intangible cultural heritage $(\mathrm{ICH})$ is a variety of traditional cultural expressions or cultural spaces that have been inherited from generation to generation by people of various ethnic groups and are closely associated to people's life. With a long history, it mirrors the cultural and artistic value, a kind of national spirit and natural heritage suitable for tourism development [1]. According to Chen (2017), the essence of the inheritance and development of ICH means a process moving from cultural capital to social and economic capital; it is not only the representation of traditional culture, but also the basic condition for the development of cultural industry [2]. As GCG, coming from Foshan, Guangdong Province, enjoys unique dyeing and finishing skills together with modern production technologies, it is possible to reduce production costs and develop distinctive tourism in line with tourism policies. Therefore, sound inheritance and development of ICH necessitates inheritors' own awareness of protection. Besides, it will be of more substantial help to its sustainable development if the 
government, industrial and educational sectors join hands with each other to spread the cultural skills of ICH.

\subsection{External Environmental Factors}

The external environment that affects the operation of an organization is complicated, and the common influencing factors include political, economic, social, cultural and technological ones. According to the past literature, the environmental factors that influence the innovative models of the inheritance of ICH include social, policy, cultural and economic ones. Social factors refer to the influences that such macro perspectives as social attitudes, foreign exchanges and local development exert on the inheritance of ICH. Policy factors mean the influences that the development strategies or policies of the government for the industry, or such environmental factors as regulations and management related to $\mathrm{ICH}$ exert on its inheritance, which constitutes the institutional foundation on which ICH depends for its survival. Cultural factors refer to the influences of local customs, cultural space, national identity and features of $\mathrm{ICH}$ activities on the inheritance of ICH [3]. Economic factors refer to the influences of a combination of production factors, industrial economic basis and economic benefits on the inheritance and innovation of ICH [4]. Among them, according to Chen (2017), cultural factors play a pivotal role in the inheritance of ICH [2]. From the analysis mentioned above, in this study, social, policy, cultural and economic factors are concluded affecting the inheritance and development of ICH of GCG in Foshan, Guangdong Province, which leads to the following hypotheses:

H1: Such external environment factors as (a) social factors, (b) policy factors, (c) cultural factors, (d) economic factors have a positive influence on the inheritance and development of GCG.

\subsection{Live Inheritance}

The driving force of external live inheritance comes from the local government, relevant enterprises, social organizations and consumers [5]. The local government has the comprehensive management functions of politics, economy, culture and society; Relevant enterprises such as manufacturers are the executors and direct participants of the development of cultural industries; Social organizations are indirect participants; Consumers are the driving force of the market demands for ICH. Thanks to the external live inheritance, it will be more helpful for the profit and sustainable development of $\mathrm{ICH}$. The driving force of internal live inheritance includes residents in the community and inheritors [5]. Community residents are the fundamental driving force of the innovative models of the inheritance of ICH; The inheritors hold the key to the inheritance of $\mathrm{ICH}$ because they have mastered the skills of ICH and play an important role in promoting the protection and inheritance of ICH. Live inheritance exerts an influence on the inheritance and development of ICH. Past studies have shown that social, policy, cultural and economic factors also have an impact on the internal and external live inheritance of $\mathrm{ICH}[2,3,5]$. Therefore, in this study, it is suggested that the external environment also has an impact on the live inheritance of GCG, which further affects the inheritance and development of GCG. The following hypotheses are as follows:

H2: (a) External live inheritance, (b) internal live inheritance have a positive impact on the inheritance and development of GCG

H3: The external environment including (a) social factors, (b) policy factors, (c) cultural factors and (D) economic factors has a positive influence on the external live inheritance of GCG

H4: The external environment including (a) social factors, (b) policy factors, (c) cultural factors and (d) economic factors has a positive influence on the internal live inheritance of GCG

H5: As the external environment including (a) social factors, (b) policy factors, (c) cultural factors and (d) economic factors has an influence on the development of GCG, external live inheritance serves as a mediator.

H6: As the external environment including (a) social factors, (b) policy factors, (c) cultural factors and (d) economic factors has an influence on the development of GCG, internal live inheritance serves as a mediator.

\section{RESEARCH METHODS}

Based on the literature mentioned above, in this study, common residents acted as the research object and questionnaires were issued and data were collected through the online survey platform. The scale of the questionnaire was designed with reference to past literature. Among them, the measurement items of social, policy, cultural and economic factors were taken from Chen's (2017) [2], while the measurement items of internal and external live inheritance and the development of GCG were referenced from Zhang \& Chen (2019)[4] and Xia's (2011)[5], together with Likert scale. The reliability analysis results of 30 valid samples pre-tested show that Cronbach's $\alpha$ of all variables was or more than 0.70 . Investigation was conducted after the reliability was confirmed.

\section{ANALYSIS RESULTS}

The effective sample of this questionnaire was 385 , with the recovery rate standing at $100 \%$, and the effective questionnaire rate $100 \%$. Among them, $65.97 \%$ were female while $34.03 \%$ were male. $70.91 \%$ of the residents 
knew about Chin's ICH; When ICH in Foshan was mentioned, $30.13 \%$ of residents thought of GCG. The results of hypothesis testing are discussed from the perspective of reliability and validity analysis and regression analysis.

\subsection{Reliability and Validity Analysis}

As can be seen from Table 1, Cronbach's $\alpha$ of all variables was more than 0.70 , while corrected item-total correlation (CITC) was more than 0.50 .

Table 1. Reliability and validity

\begin{tabular}{|c|c|c|c|c|c|c|c|}
\hline \multicolumn{2}{|c|}{ Construct \& Item } & Cronbach's a & CITC & $\begin{array}{l}\text { Factor } \\
\text { loading }\end{array}$ & $\begin{array}{c}\text { CR } \\
\text { (AVE) }\end{array}$ & $\begin{array}{c}\text { KMO } \\
\left(\text { Bartlett's } X^{2}\right)\end{array}$ & APEV $(\%)$ \\
\hline \multirow{18}{*}{$\begin{array}{c}\text { External } \\
\text { Environmental } \\
\text { Factors }\end{array}$} & EMF1 & \multirow{6}{*}{0.944} & 0.839 & 0.883 & \multirow{10}{*}{$\begin{array}{c}0.653 \\
(0.918)\end{array}$} & \multirow{18}{*}{$\begin{array}{c}0.908 \\
(5471.240)\end{array}$} & \multirow{18}{*}{84.336} \\
\hline & EMF2 & & 0.881 & 0.862 & & & \\
\hline & EMF3 & & 0.881 & 0.836 & & & \\
\hline & EMF4 & & 0.850 & 0.778 & & & \\
\hline & EMF5 & & 0.806 & 0.734 & & & \\
\hline & EMF6 & & 0.739 & - & & & \\
\hline & SF1 & \multirow{4}{*}{0.921} & 0.829 & 0.744 & & & \\
\hline & SF2 & & 0.798 & - & & & \\
\hline & SF3 & & 0.834 & - & & & \\
\hline & SF4 & & 0.808 & - & & & \\
\hline & CF1 & \multirow{4}{*}{0.950} & 0.874 & 0.899 & \multirow{4}{*}{$\begin{array}{c}0.932 \\
(0.773)\end{array}$} & & \\
\hline & CF2 & & 0.886 & 0.875 & & & \\
\hline & CF3 & & 0.888 & 0.869 & & & \\
\hline & CF4 & & 0.871 & 0.873 & & & \\
\hline & EF1. & \multirow{4}{*}{0.916} & 0.827 & 0.767 & \multirow{4}{*}{$\begin{array}{c}0.866 \\
(0.684)\end{array}$} & & \\
\hline & EF2 & & 0.878 & 0.864 & & & \\
\hline & EF3 & & 0.752 & 0.846 & & & \\
\hline & EF4 & & 0.776 & - & & & \\
\hline \multirow{9}{*}{$\begin{array}{c}\text { Live } \\
\text { Inheritance }\end{array}$} & EDI1 & \multirow{5}{*}{0.933} & 0.829 & 0.820 & \multirow{5}{*}{$\begin{array}{c}0.907 \\
(0.711)\end{array}$} & \multirow{9}{*}{$\begin{array}{c}0.865 \\
(2638.794)\end{array}$} & \multirow{9}{*}{85.591} \\
\hline & EDI2 & & 0.856 & 0.898 & & & \\
\hline & EDI3 & & 0.871 & 0.789 & & & \\
\hline & EDI4 & & 0.838 & 0.861 & & & \\
\hline & EDI5 & & 0.727 & - & & & \\
\hline & IDI1 & \multirow{4}{*}{0.911} & 0.689 & - & \multirow{4}{*}{$\begin{array}{c}0.896 \\
(0.741)\end{array}$} & & \\
\hline & IDI2 & & 0.845 & 0.835 & & & \\
\hline & IDI3 & & 0.858 & 0.907 & & & \\
\hline & IDI4 & & 0.815 & 0.839 & & & \\
\hline \multirow{5}{*}{$\begin{array}{l}\text { Inheritance \& } \\
\text { Development }\end{array}$} & IAD1 & \multirow{5}{*}{0.938} & 0.829 & 0.893 & \multirow{5}{*}{$\begin{array}{c}0.952 \\
(0.800)\end{array}$} & \multirow{5}{*}{$\begin{array}{c}0.900 \\
(1636.203)\end{array}$} & \multirow{5}{*}{80.054} \\
\hline & IAD2 & & 0.873 & 0.922 & & & \\
\hline & IAD3 & & 0.847 & 0.905 & & & \\
\hline & IAD4 & & 0.809 & 0.878 & & & \\
\hline & IAD5 & & 0.804 & 0.874 & & & \\
\hline
\end{tabular}

Note: $\mathrm{CITC}=$ corrected item-total correlation; $\mathrm{CR}=$ composite reliability; $\mathrm{AVE}=$ average variance extracted; $\mathrm{APEV}=$ accumulation percentage of explained variance

In the result of factor analysis, social and policy factors were fused into a new variable "policy management factors". Seven questions such as EMF6, SF2-4, EF4, EDI5 and Idi1 were removed because factor 
loading was less than 0.70 . The remaining test questions concerning variables were in accordance with the standard that factor loading was more than 0.70 , composite reliability (CR) was over 0.60 , and average variation extracted (AVE) was more than 0.50 , which indicated sound internal consistency and aggregated validity of each variable. In addition, the accumulation percentage of explained variance (APEV) of external environment factors, live inheritance and the inheritance and development of GCG was higher than $80 \%$, indicating that there was almost no information loss. Therefore, these test questions were of high reliability and validity for predicting the inheritance and development of GCG.

\subsection{Regression Analysis}

As can be seen from Table 2, policy management factors $(\beta=0.853, t=31.958, p<0.001)$, cultural factors $(\beta=0.560, t=13.245, p<0.001)$, economic factors $(\beta=$ $0.637, t=16.179, p<0.001)$, external live inheritance $(\beta$ $=0.799, t=25.996, p<0.001)$ and internal live inheritance $(\beta=0.832, t=29.403, p<0.001)$ had a positive influence on the inheritance and development of GCG. Thus, the H1 and the $\mathrm{H} 2$ proved to be supported. In addition, policy management factors $(\beta=0.827, t=$ $28.759, p<0.001)$, cultural factors $(\beta=0.599, t=14.652$, $p<0.001)$ and economic factors $(\beta=0.619, t=15.405, p$ $<0.001$ ) had a positive effect on the external live inheritance, so the $\mathrm{H} 3$ turned out to be supported. Similarly, policy management factors $(\beta=0.820, t=$ $28.073, p<0.001)$, cultural factors $(\beta=0.565, t=13.412$, $p<0.001)$ and economic factors $(\beta=0.557, t=13.137, p$ $<0.001)$ had a positive effect on internal live inheritance, so the H4 was supported.

From Table 2, it was shown that when the influence of the external environment factors on the inheritance and development of GCG were combined with the external lively linage, the influence coefficient of policy management factor $(\beta=0.608, t=13.501, p<0.001)$, and cultural factors $(\beta=0.127, t=3.365, p<0.001)$ and economic factors $(\beta=0.232, t=6.206, p<0.001)$ on the inheritance and development of GCG were on the decline but the influence still remained. In other words, the external live inheritance served as a mediator among those factors, so the $\mathrm{H} 5$ proved to be supported. Similarly, when the influence of the external environment factors on the inheritance and development of GCG was combined with internal live inheritance, the influence coefficient of policy management factor $(\beta=0.520, t=$ $12.415, p<0.001)$, and cultural factors $(\beta=0.132, t=$ $3.921, p<0.001)$ and economic factors $(\beta=0.251, t=$ $7.942, p<0.001)$ on the inheritance and development of GCG declined but the influence still remained. In other words, the internal live inheritance served as a mediator among those factors, so the H6 proved to be supported.
Table 2. Regression analysis

\begin{tabular}{|c|c|c|}
\hline Model & $\beta$ & Adjust $R^{2}$ \\
\hline $\mathrm{H} 1(\mathrm{a})(\mathrm{b}): \mathrm{PMF} \rightarrow \mathrm{IAD}$ & $0.853^{* * *}$ & 0.727 \\
\hline $\mathrm{H} 1$ (c): $\mathrm{CF} \rightarrow \mathrm{AD}$ & $0.560^{* * *}$ & 0.312 \\
\hline $\mathrm{H} 1(\mathrm{~d}): \mathrm{EF} \rightarrow \mathrm{AD}$ & $0.637^{* * *}$ & 0.404 \\
\hline $\mathrm{H} 2(\mathrm{a}): \mathrm{EDI} \rightarrow \mathrm{IAD}$ & $0.799^{* * *}$ & 0.637 \\
\hline $\mathrm{H} 2(\mathrm{~b}): \mathrm{IDI} \rightarrow \mathrm{AD}$ & $0.832^{* * *}$ & 0.692 \\
\hline $\mathrm{H} 3(\mathrm{a})(\mathrm{b}): \mathrm{PMF} \rightarrow \mathrm{EDI}$ & $0.827^{* * *}$ & 0.683 \\
\hline $\mathrm{H} 3(\mathrm{c}): \mathrm{CF} \rightarrow \mathrm{EDI}$ & $0.599^{* * *}$ & 0.358 \\
\hline $\mathrm{H} 3(\mathrm{~d}): \mathrm{EF} \rightarrow \mathrm{EDI}$ & $0.619^{* * *}$ & 0.381 \\
\hline $\mathrm{H} 4(\mathrm{a})(\mathrm{b}): \mathrm{PMF} \rightarrow \mathrm{IDI}$ & $0.820^{* * *}$ & 0.672 \\
\hline $\mathrm{H} 4(\mathrm{c}): \mathrm{CF} \rightarrow \mathrm{IDI}$ & $0.565^{\star * *}$ & 0.318 \\
\hline $\mathrm{H} 4(\mathrm{~d}): \mathrm{EF} \rightarrow \mid \mathrm{DI}$ & $0.557^{* \star *}$ & 0.309 \\
\hline $\mathrm{H} 5(\mathrm{a})(\mathrm{b}): \mathrm{PMF} \rightarrow \mathrm{EDI} \rightarrow \mathrm{IAD}$ & $0.608^{* * *}$ & 0.754 \\
\hline $\mathrm{H} 5(\mathrm{c}): \mathrm{CF} \rightarrow \mathrm{EDI} \rightarrow \mathrm{AD}$ & $0.127^{* * *}$ & 0.647 \\
\hline $\mathrm{H} 5(\mathrm{~d}): \mathrm{EF} \rightarrow \mathrm{EDI} \rightarrow \mathrm{IAD}$ & $0.232^{* * *}$ & 0.670 \\
\hline $\mathrm{H} 6(\mathrm{a})(\mathrm{b}): \mathrm{PMF} \rightarrow \mid \mathrm{DI} \rightarrow \mathrm{IAD}$ & $0.520^{* * *}$ & 0.780 \\
\hline $\mathrm{H} 6(\mathrm{c}): \mathrm{CF} \rightarrow \mathrm{IDI} \rightarrow \mathrm{IAD}$ & $0.132^{* * *}$ & 0.703 \\
\hline $\mathrm{H} 6(\mathrm{~d}): \mathrm{EF} \rightarrow \mathrm{IDI} \rightarrow \mathrm{IAD}$ & $0.251^{* * *}$ & 0.735 \\
\hline
\end{tabular}

Note: ${ }^{*} p \leq 0.05 ; * * p \leq 0.01 ; * * * p \leq 0.001$

\section{CONCLUSION}

It is found that among the external environmental factors affecting $\mathrm{ICH}$, policy management factors (the combination of social and policy factors), cultural factors, economic factors and lively lineage have a positive impact on the inheritance and development of intangible cultural heritage of Gambiered Canton Gauze in Foshan, Guangdong Province, China. Among them, external and internal live inheritance not only have a positive effect on the inheritance and development of GCG in terms of the external environment. In other words, when policy, cultural and economic factors have greater influence on the internal and external live inheritance of $\mathrm{ICH}$, the inheritance and sustainable development of ICH can be enhanced. Therefore, following suggestions for the inheritance and development of ICH are proposed:

First, external and internal live inheritance are equally important to ICH. In terms of external live inheritance, several measures can be carried out by building better ICH base, establishing ICH museums, increasing publicity via new media, and developing cultural and creative products. In terms of internal live inheritance, it is helpful for the sustainable development of $\mathrm{ICH}$ by enlarging the inheritors' own family, community or developing cultural tourism.

Second, various external environmental factors affect the live inheritance and sustainable development of $\mathrm{ICH}$, including policy management, culture and economic factors. In terms of policy management, residents believe 
that in addition to providing better infrastructure and inheritance management, it is important to pay attention to the protection of cultural environment, the market order and the social awareness. Besides, people believe that cultural factors such as knowledge publicity, protection of resources and diversity of activities, as well as the economic factors such as whether ICH can drive local economy, increase local income and promote local employment are also very important for the inheritance and development of ICH. Therefore, it is necessary for the local government to intervene, and the joint efforts from the cooperation of industry, government and education can make the ways of inheritance of ICH more effective and practical.

\section{ACKNOWLEDGMENT}

This paper is the periodical report of the key project of Guangdong Province, Investigation and Research on the Inheritance and Development of Intangible Cultural Heritage of Gambiered Canton Gauze -- A Case Study of Foshan City, Guangdong Province (PDJH2020A0737) and the university project of innovation training, Purchasing Intention of Gambiered Canton Gauze as National Intangible Cultural Heritage - (2020CXXL033).

\section{REFERENCES}

[1] D. Tian, Q Wang, R. Law, M. Zhang, Influence of cultural identity on tourists' authenticity perception, tourist satisfaction, and traveler loyalty, Sustainability, vol. 12, MDPI, Open Access Journal, 2020, pp. $1-18$. DOI: http://doi.org/10.3390/su12166344

[2] W. Chen, Influencing factors of intangible cultural heritage inheritance of ethnic minority villages in Guangxi: based on stakeholder theory, Social Scientist, CSCD, Guangxi, China, 2017, no. 01, pp. 96-102. DOI: https://doi.org/10.3969/j.issn.10023240.2017.01.017

[3] C. X. Zhang, L. H. N. Fong, S. N. Li, T. P. Ly, National identity and cultural festivals in postcolonial destinations, Tourism Management, Elsevier, 2019, vol. 73, pp. 94-104. DOI: https://doi.org/10.1016/j.tourman.2019.01.013

[4] L. Zhang, W. Chen, Construction of inheritance and innovation mode of intangible cultural heritage of ethnic minority villages in Guangxi, Journal of Guilin University of Technology, CSCD, Guangxi, China, 2019, vol. 39, no. 01, pp. 241-248. DOI: https://doi.org/ $10.3969 /$ j.issn. 1674 9057.2019.01.031

[5] N. Xia, Research on the inheritance of intangible cultural heritage, Master Thesis, Yunnan: Yunnan Institute of Art, 2011. 